WestVirginiaUniversity
THE RESEARCH REPOSITORY @ WVu

Faculty Scholarship

1997

\title{
A mass resolving neutral atom imager
}

Earl E. Scime

Paul A. Keiter

Follow this and additional works at: https://researchrepository.wvu.edu/faculty_publications

\section{Digital Commons Citation}

Scime, Earl E. and Keiter, Paul A., "A mass resolving neutral atom imager" (1997). Faculty Scholarship. 33.

https://researchrepository.wvu.edu/faculty_publications/33

This Article is brought to you for free and open access by The Research Repository @ WVU. It has been accepted for inclusion in Faculty Scholarship by an authorized administrator of The Research Repository @ WVU. For more information, please contact ian.harmon@mail.wvu.edu. 


\title{
A mass resolving neutral atom imager
}

\author{
Earl E. Scime and Paul A. Keiter \\ Department of Physics, West Virginia University, Morgantown, West Virginia 26506
}

(Presented on 13 May 1996)

\begin{abstract}
Neutral atom imaging of space plasmas, the measurement of neutral atom flux as a function of angle and energy, can provide qualitative information about the global dynamics of a space plasma. In addition, mass and energy resolved neutral atom images can be used to remotely measure convective electric fields in planetary magnetospheres. Direct detection of neutral atoms using ultraviolet-rejecting waveguides and microchannel plate sensors has been proposed as a method of imaging low-energy neutral atoms. We discuss a new type of direct detection neutral atom imager that is capable of producing mass and energy resolved images. The imager has a large geometric factor $\left(G \approx 0.2 \mathrm{~cm}^{2} \mathrm{sreV} / \mathrm{eV}\right)$, an energy range of $1-100 \mathrm{keV}$, and single coincidence noise suppression. The estimated mass of the imager is less than $1.5 \mathrm{kG}$. (C) 1997 American Institute of Physics. [S0034-6748(97)51501-2]
\end{abstract}

\section{INTRODUCTION}

With the launch of the first neutral atom imager aboard the ASTRID spacecraft in $1995,{ }^{1}$ the race to image a planetary magnetosphere by neutral atom emission has begun. Hampered by a faulty spacecraft power system, the PIPPIE neutral atom imager aboard ASTRID operated long enough to record $8 \mathrm{~h}$ of high-energy neutral atom flux and $5 \mathrm{~h}$ of low-energy neutral atom flux. ${ }^{1}$ The next chance for neutral atom imaging appears to be the Cassini mission to Saturn. Cassini is scheduled to launch in 1997 and arrive at Saturn in 2003. Neutral atoms with energies greater than $20 \mathrm{keV}$ will be imaged using an advanced energetic particle telescope. ${ }^{2}$ Instruments capable of imaging lower-energy neutrals have been proposed. ${ }^{3-5}$ As computer simulations ${ }^{6}$ based on in situ measurements predict magnetospheric neutral fluxes of 100$1000 \mathrm{~cm}^{-2} \mathrm{~s}^{-1} \mathrm{sr}^{-1}$ and measured geocoronal ultraviolet photon fluxes ${ }^{7}$ are $10^{9} \mathrm{~cm}^{-2} \mathrm{~s}^{-1} \mathrm{sr}^{-1}$, the key issues for lowenergy neutral atom imaging are sensitivity and photon background rejection. A low-energy neutral atom imager must have a large geometric factor (throughput) and substantial photon background rejection.

Neutral atom detection techniques can be divided into two categories: indirect detection and direct detection. Indirect detection relies on a modification of the charge state of the neutral atom before detection. This is accomplished by either stripping an electron from ${ }^{4}$ or adding an electron to ${ }^{3}$ the neutral atom. The ionized neutral can then be energy analyzed and imaged with a standard electrostatic analyzer (Fig. 1). If the charge modification process is handled carefully, the trajectory of the incident neutral can be determined upon final detection. Photon background is eliminated by conveying the ionized neutral atoms into a detection region which is blocked from direct access by photons (e.g., in Fig. 1 the photons cannot make it around the hemispherical analyzer). Direct detection was first proposed by Gruntman in $1991{ }^{8}$ Essentially, the neutral atoms fall directly onto a sensitive detector while photons are eliminated by a filter that permits the passage of atoms but not ultraviolet photons (Fig. 2). Recent measurements of 2000- $\AA$-period, free-standing gold gratings suggest that it is possible to construct a direct detection neutral atom imager using the gratings as ultraviolet filters. ${ }^{9}$ The biggest drawback to direct detection techniques has been the lack of mass information in the final image. When the typical postionization analysis method for indirect detection techniques (energy per charge electrostatic analysis) is combined with a time-of-flight (TOF) detector, determination of the neutral atom's mass is straightforward. ${ }^{10}$ In this article, we describe a direct detection technique that provides mass identification while maintaining the large geometric factor and high background rejection level characteristic of a direct detection imager.

\section{INSTRUMENT DESIGN}

Fundamentally, a direct neutral atom imager is simply a neutral-sensitive detector placed behind an ultraviolet photon rejecting, particle passing filter (Fig. 2). In order to determine the incident neutral atom trajectory and improve the signal-to-noise level of the instrument, a thin carbon foil is placed just behind the filter. Secondary electrons generated at the rear surface of the foil during the passage of neutral atoms are focused onto a nearby detector with a strong electric field. The electron's impact location corresponds to the point at which the neutral atom passed through the entrance aperture. Every electron detection event also triggers a system of TOF electronics. When the neutral atom strikes another position-sensitive detector it generates a stop pulse for the TOF system and the position information needed to determine the incident neutral atom's trajectory and velocity.

Either the mass is needed to calculate the neutral atom's energy, or the energy is needed to calculate the mass. Indirect detection techniques (as shown in Fig. 1) can measure the mass per unit charge because the neutral atoms are energy per charge analyzed after they are ionized. By taking advantage of the fraction of neutrals that become ionized during passage through the thin carbon foil and the substantial difference in the polarity of the exit charge states for hydrogen and oxygen neutrals, ${ }^{11}$ it is possible to build a direct detection neutral atom imager that provides both energy and mass information. Although previous theoretical studies have assumed that the neutral atom flux from the inner magneto- 


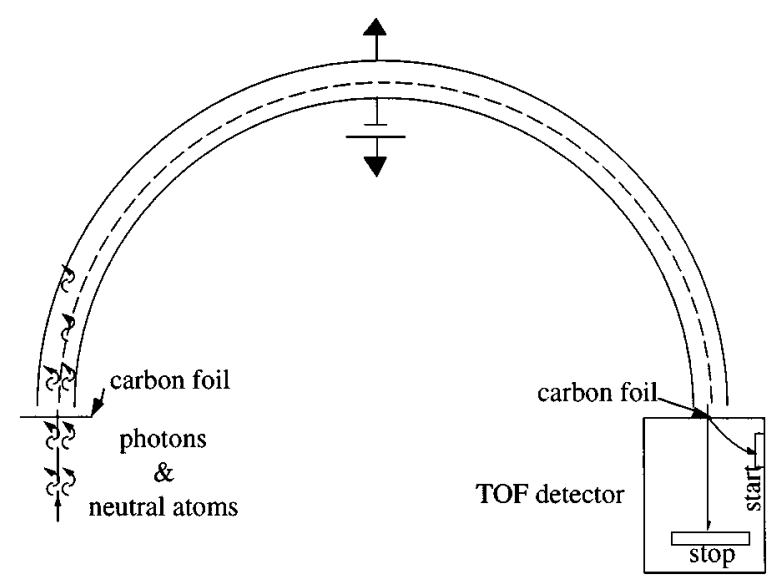

FIG. 1. A direct detection neutral atom detector (see Ref. 4). Neutral atoms are ionized at the entrance with a thin carbon foil $(40 \AA)$ and directed to a TOF detector. Ultraviolet photons pass through the carbon foil but are prevented from reaching the TOF detector by the $180^{\circ}$ electrostatic analyzer. With the $E / q$ information from the analyzer and the velocity information from the TOF detector, the mass per unit charge of the incident neutrals can be determined.

sphere will be dominated by hydrogen and oxygen, ${ }^{6,12}$ recent AMPTEE spacecraft measurements suggest the possibility of a significant helium component during intense substorm periods. ${ }^{13}$ Therefore, a mass-resolving neutral atom imager must be capable of discriminating between hydrogen, helium, and oxygen neutrals.

The operational principal of the new imager is depicted in Fig. 3. Collimated fans (to within $\pm 4^{\circ}$ ) of neutral atoms pass through a 5000- $\AA$-thick, free-standing 1000- $\AA$-period gold grating. The grating is aligned so that the long slots of the grating are perpendicular to the $\pm 4^{\circ}$ collimation. The measured ratio of particle to ultraviolet transmission of a single 2000-Å-period grating is approximately $10^{4} .{ }^{9}$ Numerical models benchmarked with data from the $2000-\AA$-period gratings predict that a $1000-\AA$-period grating will have a relative transmission of $10^{7}$, more than enough to reduce the flux of ultraviolet photons to a level manageable by singlecoincidence particle detection. 1000- $\AA$-period gratings supported by a polymid membrane are now routinely manufactured $^{14}$ and preliminary free-standing versions have

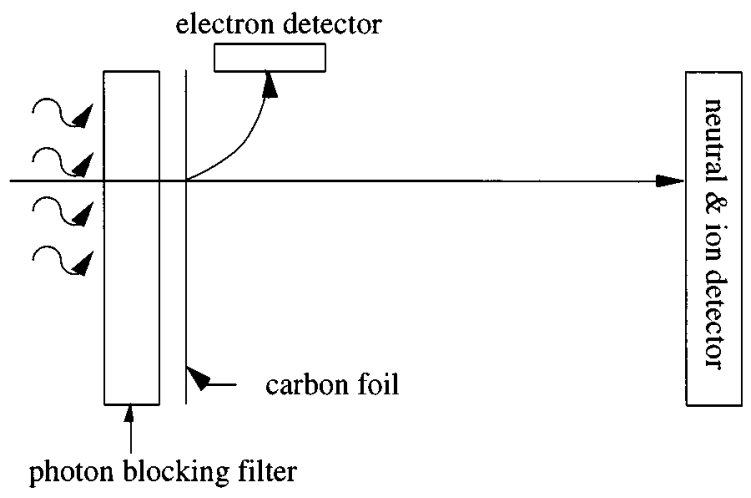

FIG. 2. Direct detection technique including TOF measurement.

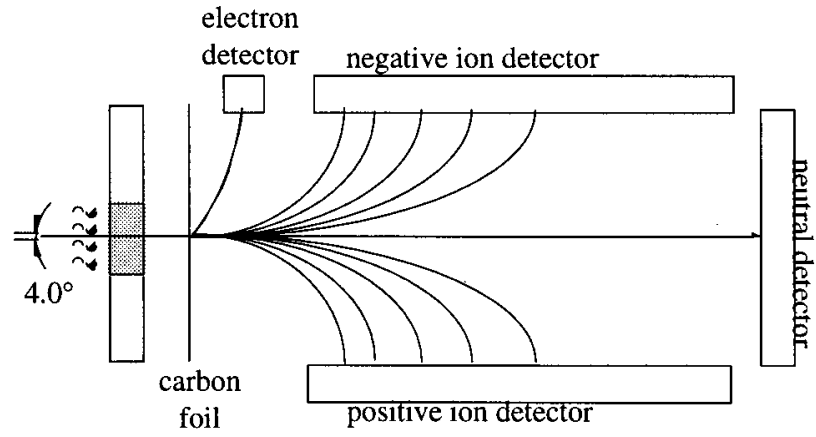

FIG. 3. Operational schematic of mass resolving direct neutral atom detector. Collimated beams of neutral atoms pass through a photon blocking gold grating and transit a thin carbon foil. Secondary electrons are focused onto the start detector for TOF and position measurements. Negatively (positively) charged ions strike the upper (lower) position-sensitive detectors.

been constructed (M. L. Schattenburg, private communication). After passing through the grating, the neutral atoms pass through a thin, $40-\AA$-thick carbon foil. The majority of the atoms remain neutral and strike the rear positionsensitive microchannel plate detector. Depending on their energy, some of the neutrals become ionized during passage through the foil. ${ }^{11}$ Hydrogen and helium atoms are preferentially positively ionized, while oxygen atoms are preferentially negatively ionized.

The trajectories of those neutrals which become ionized bend in the electric field applied across the imager $(+10 \mathrm{kV}$ on upper detector, $-10 \mathrm{kV}$ on lower). Since their final impact point is a function of the incident neutral energy, the microchannel detectors along the top and the bottom function as energy per charge spectrographs. The trajectory of all the incident neutral atoms is determined by the location of the start pulse along the thin carbon foil and the location of the final stop pulse. The key features of this imager include: all of the incident neutrals become detectable events (boosting sensitivity); secondary electrons from the foil are focused to a separate detection area (permitting rapid and unique coincidence/trajectory determinations); the energy range is determined by the TOF electronics; and oxygen is crudely separated from hydrogen and helium by the disparity in exit charge states. Essentially three images are produced by this instrument. The microchannel plate detector along the rear generates a velocity resolved image of all incident neutral atoms. The upper microchannel plate generates a mass resolved image of negatively ionized neutrals (primarily oxygen). The lower microchannel plate image duplicates the upper for positively charged ions (primarily hydrogen and helium). For compactness, the upper microchannel plate can also serve as the electron detector. Thus, the entire upper surface of the instrument can be kept at the same potential.

\section{ELECTROSTATIC SIMULATIONS}

A fully three-dimensional electrostatic model of the imager [Fig. 4(a)] was constructed with the SIMION 6.0 computer code. ${ }^{15}$ The objective, as with all space-based instruments, is to maximize the geometric factor while minimizing the size 
of the imager. In order to use the entire aperture, all secondary electrons emitted from the carbon foil must be accurately focused onto the upper microchannel plate detector. To approximate actual secondary electron distributions, ${ }^{16}$ the secondary electron energy is chosen randomly between 1 and 10 $\mathrm{eV}$ and the emission is assumed to be isotropic from a point source on the foil. Figure 4(b) demonstrates that regardless of their emission angle and energy, electrons from all four corners and the center of the carbon foil are uniquely focused onto the upper detector. The $20 \mathrm{kV}$ potential difference across the upper and lower microchannel plates is sufficient to strongly focus all of the secondary electrons onto the upper detector. Only a one-dimensional position readout for the electron portion of the upper detector is needed to determine entrance point of the neutral atom along the length of the carbon foil.

Figure 4(c) shows that emitted electrons from two points on the foil separated by $3 \mathrm{~mm}$ are distinguishable at the electron detector. When combined with the position information from the rear detector [Fig. 4(a)], the incident neutral atom trajectory can be resolved to within $2^{\circ}$. Scattering in the foil, however, goes as ${ }^{17}$

$$
12.6^{\circ} \mathrm{keV} / E_{\text {neutral }},
$$

so the intrinsic angular resolution becomes important for $E_{\text {neutral }} \geqslant 6 \mathrm{keV}$.

Since the neutral atoms are collimated to within $\pm 4^{\circ}$ along the short axis of the aperture, the electron detector need only be one dimensional. The simulation indicates that the arrival time spread of the secondary electrons emitted from a single point on the foil is less than $0.6 \mathrm{~ns}$. This intrinsic timing spread limits TOF energy measurements to less then a few $\mathrm{MeV}$ for neutral hydrogen.

The one-dimensional microchannel plate detector along the rear of the instrument provides the end point of the trajectory for neutrals that exit the foil as neutrals or highenergy ions [energy above $55 \mathrm{keV}$, see Fig. 4(a)]. Therefore, the rear detector is responsible for imaging roughly $80 \%$ of the incident neutral atoms with energy below $55 \mathrm{keV}$ and all of the incident neutrals with energies above $55 \mathrm{keV}$. The coincidence window of the TOF electronics will prevent start or stop pulses generated by background photons from being classified as neutral atom events (a photon can produce a start or a stop pulse, but not both).

Data from the electron and rear microchannel plates can be used to generate a velocity resolved image of the neutral atom flux incident along the long axis of the aperture. The angular acceptance along the aperture varies from $+32^{\circ}$ and $-72^{\circ}$ at the edge of the aperture to $\pm 62^{\circ}$ in the center. By placing the imager aboard a spinning spacecraft, the $\pm 4^{\circ}$ collimation along the short axis of the entrance aperture will sweep out the entire $360^{\circ}$ rotation with $4^{\circ}$ resolution. Total angular coverage is approximately $60 \%$ of the $4 \pi$ sr unit sphere.

Those atoms which become positively (negatively) ionized in the start foil will be attracted to the bottom (top) of the instrument. The $20 \mathrm{keV}$ potential difference across the instrument not only cleanly focuses the electrons onto the part of the upper detector closest to the foil, but also serves
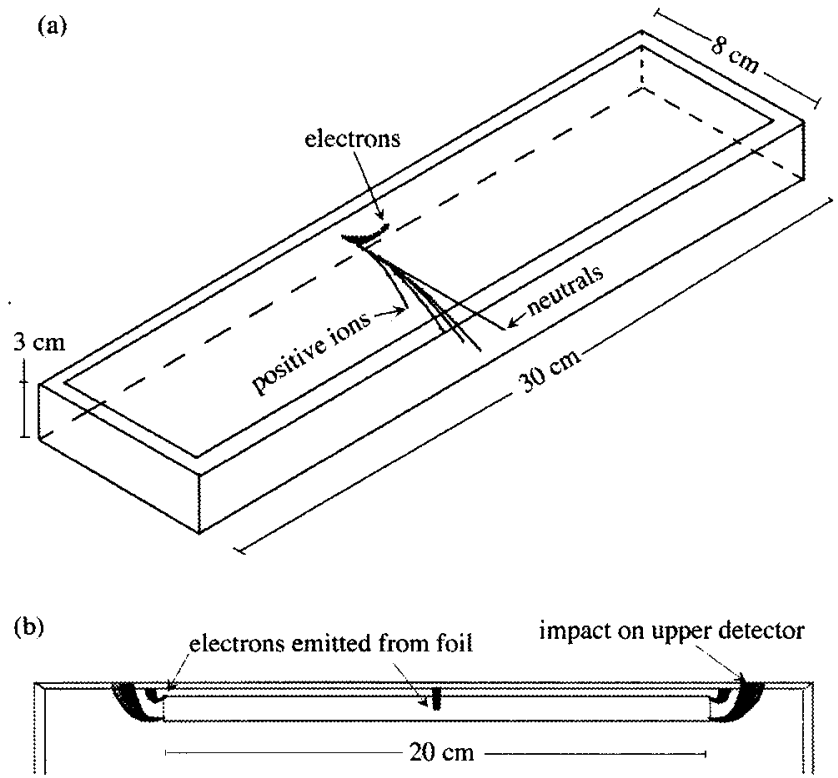

(c)

(d)
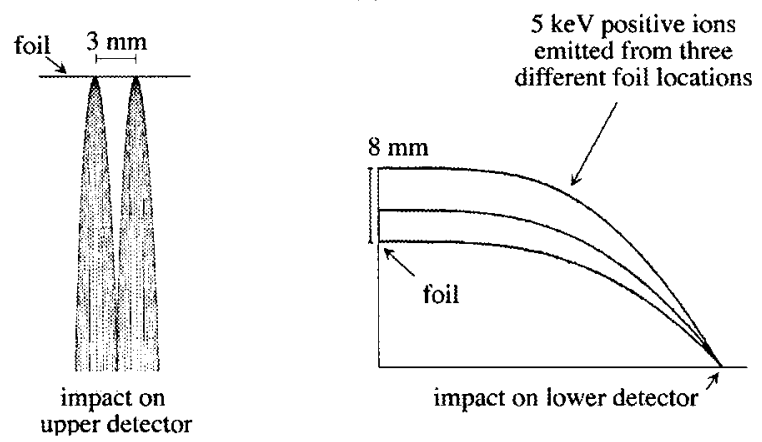

FIG. 4. Different views from the electrostatic simulation of the massresolving, direct detection neutral atom imager. (a) Overall view showing incident neutrals, straight-through neutrals, start electrons being focused onto the upper microchannel plate detector, and an energy spectrogram of positively charged "ionized neutrals" being imaged onto the lower detector. (b) View showing that electrons from entire foil are focused onto electron detector. (c) Enlarged view showing start position resolution attainable with electron detector. Electrons are launched from foil and impact on upper detector. (d) View showing different positively ionized neutral trajectories that impact the same point on lower detector.

to spread out the positive and negative ion trajectories as a function of incident energy [Fig. 5(a)]. Determination of the incident neutral atom trajectory and mass requires a twodimensional, position-sensitive readout behind the upper and lower microchannel plate detectors. ${ }^{18}$ The position along the long axis of the aperture provides the angular information and the position between the aperture and the rear detector provides a measurement of the energy per charge (once the trajectory is known). Very low-energy negative ions can land on the electron portion of the upper detector, but will not result in an event because coincidence is required between an electron region start pulse and a stop pulse from one of the other three detector regions.

The start and stop pulses also yield the velocity of the atom once the trajectory is estimated. Assuming the "ionized" neutral is singly charged, the mass of the neutral can 
be determined from the energy and velocity information. Only crude mass resolution $(m / \Delta m \approx 1.3)$ is necessary to separate hydrogen, helium, and oxygen. The mass resolution is given by

$$
\frac{m}{\Delta m}=\left[\left(\frac{\Delta E}{E}\right)^{2}+4\left(\frac{\Delta d}{d}\right)^{2}+4\left(\frac{\Delta t}{t}\right)^{2}\right]^{-1 / 2},
$$

where $E$ is the energy corresponding to a particular stop position, $d$ is the path length, and $t$ is the TOF interval. Figure 4(d) shows the spread in initial positions that lead to the same final position for positively ionized neutrals with a nominal energy of $5 \mathrm{keV}$. Each trajectory corresponds to a slightly different energy, thus, Fig. 4(d) is reflective of the intrinsic energy and trajectory measurement error of the imager. For Fig. 4(d), the path length and energy spreads are $16 \%$ and $31 \%$, respectively. Conservatively estimating a TOF error of $10 \%$ gives a mass resolution $m / \Delta m$ of approximately 2.0 .

Once the relationship between mass, velocity, and incident angle is determined with the upper and lower detectors, velocity and spatial "bands" from the more sensitive "straight-through" detection process can be used to provide enhanced information about those regions. For example, if neutral atom emission from the magnetotail is dominated by hydrogen atoms, the straight-through detector data corresponding to that look direction can be assumed to be entirely hydrogen and be used for higher time resolution measurement of plasma convection in the magnetotail.

\section{EXPECTED PARAMETERS}

Given the instrument parameters simulated in Fig. 4, a $0.8 \times 20 \mathrm{~cm}^{2}$ aperture, overall dimensions of $30 \times 8 \times 3 \mathrm{~cm}^{3}$, and collimation of $\pm 4^{\circ}$, the approximate mass and geometric factor $G$ of the imager can be calculated,

$G=\epsilon_{\mathrm{mcp}} \epsilon_{\mathrm{TOF}} T w \int_{-4^{\circ}}^{4^{\circ}} d \phi \int_{-10 \mathrm{~cm}}^{10 \mathrm{~cm}} d x \int_{-\theta_{2}(x)}^{\theta_{1}(x)} \cos \theta d \theta$,

where the efficiency of the microchannel plate detector $\epsilon_{\mathrm{mcp}}$ is 0.90 ; the efficiency of the TOF system, $\epsilon_{\mathrm{TOF}}$ is 0.65 ; the transparency of the grating $T$ is 0.15 ; the width of the aperture $w$ is $0.8 \mathrm{~cm}$; and the energy bin width $(\Delta E / E)$ determined by the choice of time window for the TOF electronics is assumed to be unity. These parameters give a geometric factor of

$$
G=0.2 \mathrm{~cm}^{2} \mathrm{sr} \mathrm{eV} / \mathrm{eV} .
$$

For a $5 \mathrm{keV}$ neutral flux of 100 per $\mathrm{cm}^{-2} \mathrm{~s}^{-1} \mathrm{sr}^{-1}$, the instrument would register 20 counts per second. It is important to note that the count rate at any given time is considerably larger as all energies are sampled simultaneously. This count rate is large enough for high-time resolution images of the near Earth magnetosphere, the plasma sheet, and perhaps the magnetotail. ${ }^{6}$

A conservative estimate for the mass of just the imaging assembly, including microchannel plates, is $1.5 \mathrm{kG}$. Allotting $3.5 \mathrm{kG}$ for the power supplies, TOF electronics, and the collimator, the entire instrument should mass about $5 \mathrm{kG}$.

\section{CONCLUSIONS}

The electrostatic simulation described in this article suggests that it is possible to build a compact, lightweight, massresolving, high-sensitivity neutral atom imager. The results of the simulation indicate that the imager (not including the electronics, power supplies, or collimator) will have an energy range of $1-100 \mathrm{keV}$, a total mass of $1.5 \mathrm{kG}$, an intrinsic angular resolution of $1.5^{\circ} \times 4^{\circ}$, a geometric factor of 0.2 $\mathrm{cm}^{2} \mathrm{sr} \mathrm{eV/eV}$, and a mass resolution of 1.4. We note that a recently proposed, mass-resolving, indirect imager with a geometric factor of $0.4 \mathrm{~cm}^{2} \mathrm{sr}^{1}$ for $10^{\circ} \times 10^{\circ}$ angular resolution $\left(0.02 \mathrm{~cm}^{2} \mathrm{sr}^{1}\right.$ for $2^{\circ} \times 2^{\circ}$ resolution) and an energy range of $1-40 \mathrm{keV}$ was estimated to mass $10 \mathrm{kG} .^{19}$

Facilities for construction and testing of a prototype imager based on the electrostatic simulation are under development at West Virginia University.

\section{ACKNOWLEDGMENTS}

This work was supported by a grant from NASA through the West Virginia Space Grant Consortium. Permission from Dave Dahl to use a preliminary version of the SIMION electrostatic code is gratefully acknowledged.

${ }^{1}$ O. Norberg, S. Barabash, I. Sandahl, R. Lundin, H. Lauche, H. Koskinen, P. C. Brandt, E. Roelof, L. Andersson, U. Eklund, H. Borg, J. Gimholt, K. Lundin, J. Rynö, and S. Olsen, in Proceedings of the 12th ESA Symposium on European Rocket and Balloon Programmes and Related Research, Lillehammer, Norway, 1995 (unpublished).

${ }^{2}$ D. G. Mitchell, A. F. Cheng, S. M. Krimigis, E. P. Keath, S. E. Jaskulek, B. H. Mauk, R. W. McEntire, E. C. Roelof, D. J. Williams, K. C. Hsieh, and V. A. Drake, Opt. Eng. 32, 3096 (1993).

${ }^{3}$ A. G. Ghielmetti, E. G. Shelly, S. A. Fusilier, P. Wurz, P. Bochsler, F. A. Herrero, M. F. Smith, and T. S. Stephen, Opt. Eng. 33, 362 (1994).

${ }^{4}$ D. J. McComas, B. L. Barraclough, R. C. Elphic, H. O. Funsten, and M. F. Thomsen, Proc. Natl. Acad. Sci. USA 88, 9598 (1991).

${ }^{5}$ E. E. Scime, H. O. Funsten, M. A. Gruntman, and D. J. McComas, Opt. Eng. 33, 357 (1994).

${ }^{6}$ K. R. Moore, E. E. Scime, H. O. Funsten, D. J. McComas, and M. F. Thomsen, Opt. Eng. 33, 342 (1994).

${ }^{7}$ K. C. Hseih, E. Keppler, and G. Schmidtke, J. Appl. Phys. 5, 2242 (1980).

${ }^{8}$ M. A. Gruntman, Proc. SPIE 1549, 385 (1991).

${ }^{9}$ E. E. Scime, E. H. Anderson, D. J. McComas, and M. L. Schattenburg, Appl. Opt. 34, 648 (1995).

${ }^{10}$ D. J. McComas, H. O. Funsten, J. T. Gosling, K. R. Moore, E. E. Scime, and M. F. Thomsen, Opt. Eng. 33, 335 (1994).

${ }^{11}$ H. O. Funsten, D. J. McComas, and B. L. Barraclough, Opt. Eng. 32, 3090 (1993).

${ }^{12}$ M. Hesse, M. F. Smith, F. Herrero, A. G. Ghielmetti, E. G. Shelley, P. Wurz, P. Bochsler, D. L. Gallagher, T. E. Moore, and T. S. Stephen, Opt. Eng. 32, 3153 (1993).

${ }^{13}$ S. A. Fuselier, D. M. Klumpar, and E. G. Shelley, J. Geophys. Res. 96, 21095 (1991).

${ }^{14}$ T. A. Savas, S. N. Shah, M. L. Schattenburg, J. M. Carter, and H. I. Smith, J. Vac. Sci. Technol. B 13, 2732 (1995). (unpublished).

${ }^{15}$ Developed by Dave Dahl, Idaho National Engineering Laboratory, Idaho Falls, ID 83415.

${ }^{16}$ F. J. Pijper and P. Kruit, Phys. Rev. B 44, 9192 (1991).

${ }^{17}$ H. O. Funsten, D. J. McComas, and E. E. Scime, J. Spacecraft and Rockets 32, 899 (1995).

${ }^{18}$ See, for example, Ref. 2.

${ }^{19}$ B. H. Mauk et al., NASA Medium-Class Explorer Mission (MIDEX) proposal, 1995. 